\title{
BAP (PAH) air quality modelling exercise over Zaragoza (Spain) using an adapted version of WRF-CMAQ model \\ R. San Jose ${ }^{* 1}$, J.L. Perez ${ }^{1}$, M. Callen ${ }^{2}$, J.M. Lopez ${ }^{2}$, A. Mastral ${ }^{2}$ \\ ${ }^{1}$ Echnical University of Madrid (UPM), Spain, ${ }^{2}$ ICB-CSIC, Spain
}

$\mathrm{BaP}$ is one of the most toxic pollutants which are present in the atmosphere, BaP is a polycyclic aromatic hydrocarbon (PAH) which are environmental pollutants of transboundary character. They are products of the incomplete combustion independently of the used fuel and they have a great impact on human health. They can be originated by biogenic emission sources like volcanoes or forest fire but the main sources are related with anthropogenic sources like power generation, transport systems and residential combustion. Into the PAH family, the Benzo(a)pyrene (BaP) is one of the most dangerous PAH due to its high carcinogenic and mutagenic character. Because of this reason, the Directive 2004/107/CE of the European Union establishes a target value of $1 \mathrm{ng} / \mathrm{m} 3$ of $\mathrm{BaP}$ in the atmosphere.

In this paper, the main aim is to estimate the BaP concentrations in the atmosphere by using last generation of air quality dispersion models with the inclusion of the transport, scavenging and deposition processes for the BaP. To reach this aim, a detailed emission inventory has been developed. $\mathrm{BaP}$ is injected into the atmosphere as particle and the degradation of the particulated $\mathrm{BaP}$ by the ozone has been considered. The aerosol-gas partitioning phenomenon in the atmosphere is modeled taking into a count that the concentrations in the gas and the aerosol phases are in equilibrium.

The model has been validated in the area of Zaragoza (Spain) during 10 weeks in 2010. A validation process of the $\mathrm{BaP}$ results obtained with the model at local scale in the atmosphere of the Zaragoza city has been conducted. The agreement is generally satisfactory with important influence of the meteorological conditions.

Keywords: BaP, PAH, AIR QUALITY, SIMULATION 